\title{
COMPARATIVE STUDY OF ORAL HYPOGLYCEMIC AGENTS IN TYPE-2 DIABETES OBESE PATIENTS
}

\author{
VENKATESWARLU KONURU ${ }^{1 *}$, RAM MOHAN REEDY T $^{2}$ \\ ${ }^{1}$ Research Scholar, Department of Pharmacy, Mewar University, Chittorgarh, Rajasthan, India. ${ }^{2}$ Research Guide, Department of Pharmacy, \\ Mewar University, Chittorgarh, Rajasthan, IndiaEmail: venkipharmd@gmail.com
}

Received: 12 February 2018, Revised and Accepted: 10 March 2018

\section{ABSTRACT}

Objectives: The aim of this study was to evaluate safety and efficacy of oral hypoglycemic agents in obese Type-2 diabetic patients. The objectives are to compare fasting and postprandial blood sugar (PPBS) levels, to compare body mass index (BMI) in all the groups, and to identify glycosylated hemoglobin levels and adverse drug reactions (if present) in all the groups.

Method: This is a prospective observational study conducted in care diabetic center over a period of 1 year. All the patients those are receiving only oral hypoglycemic agents continuously over a period of 3 months and BMI $\geq 30$ were enrolled. The patients receiving insulin were excluded. Patients were followed over a period of 3 months and were reviewed on visit basis (every 30 days). All the necessary information was collected into the data collection form that includes demographic details (age, gender, etc.), past medication history, current treatment charts, and their relevant laboratory reports (fasting blood sugar levels [mg/dl], PPBS levels [mg/dl], glycosylated hemoglobin A1c [HbA1c] (\%), and BMI [kg/m²]).

Results: A total of 395 patients were recruited into the study and the drugs received by the population were found to be metformin+sulfonylureas (33\%), metformin+pioglitazone (26\%), and metformin+dipeptidyl peptidase inhibitors (DPI) (23\%). A significant reduction in HbA1c was seen in all groups of patients. Adverse drug reactions observed were hypoglycemia, pedal edema, and itching distributed to drugs metformin+DPI, respectively. A significant reduction in BMI was seen in patients receiving DPI and BMI was found to be increased in other groups of patients.

Conclusion: Overall, three classes of drugs were found to have similar efficacy. Sulfonylureas were commonly associated with hypoglycemia when compared to other drugs and weight reduction observed in dipeptidyl peptidase inhibitors.

Keywords: Type-2 Diabetes, Obesity Patients, oral hypoglycaemic Agents.

(C) 2018 The Authors. Published by Innovare Academic Sciences Pvt Ltd. This is an open access article under the CC BY license (http://creativecommons. org/licenses/by/4. 0/) DOI: http://dx.doi.org/10.22159/ajpcr.2018.v11i6.25264

\section{INTRODUCTION}

Defective insulin secretion and insulin resistance appear very too early in obese patients, and both worsen equally as diabetes progresses. An increase in overall fat tissue, especially in visceral as well as ectopic fat depots, is particularly associated with insulin resistance. The relationship between obesity and diabetes is of such interdependence that the term "diabesity" has been coined [1]. The prevalence of diabetes for all age groups worldwide was estimated to be $2.8 \%$ in 2000 and it will be $4.4 \%$ in 2030 . The total number of people with diabetes is projected to rise from 171 million in 2000-366 million in 2030. Obesity is one of the modifiable risk factors of diabetes which has its profound effects on the glucose levels the diabetic patient [2]. A lifestyle modification to lose weight is recommended for diabetic patients to improve glycemic control and diminish-associated risk factors of microvascular and macrovascular complications [3]. Even modest weight loss can appreciably lessen glucose levels and decrease cardiometabolic risk factors. However, many antidiabetic drugs such as insulin, sulfonylureas, and thiazolidinediones are associated with weight gain, making treatment of overweight or obese patients with type 2 diabetes quite challenging. However, incretin-based therapies with the new classes of glucagon-like peptide- 1 mimetics (e.g., exenatide, liraglutide) and dipeptidyl peptidase 4 (DPP-4) inhibitors (e.g., sitagliptin, vildagliptin) may be of particular value in the treatment of overweight/obese type 2 diabetic patients because of their efficacy in improving glycemic control and their favorable or neutral effects on body weight [4]. The previous study suggested that combination of treatment with metformin plus glimepiride was more effective in improving lipid status of Indian type 2 diabetic patients and many studies have shown that their is positive correlation between lipid levels and body weight [5]. In addition, DPP-4 inhibitors have a low risk of causing hypoglycemia, undesirable gastrointestinal effects, or other prominent adverse effects that might limit their use. These classes of drugs hold promise for the treatment of type 2 diabetes, alone or in combination with other classes of antidiabetic agents [6]. As it is important to evaluate the benefits of hypoglycemic agents in patients with various confounding risk factors, a comparative study is advantageous to choosing a right drug for the obese patient to reduce weight or put weight in control. The aim of this study was to evaluate safety and efficacy of oral hypoglycemic agents in obese Type-2 diabetic patients. The objectives are to compare fasting and postprandial blood sugar (PPBS) levels, to compare body mass index (BMI) in all the groups and to identify glycosylated hemoglobin levels and adverse drug reactions (if present) in all the groups.

\section{METHODOLOGY}

A prospective observational study was performed at care diabetes center in Telangana region after getting approval from the Ethical Committee for a period of 1 year. Patients with type 2 diabetes mellitus of both sexes and age group $>18$ years and are on oral hypoglycemic agents continuously over a period of 3 months, with BMI 30 were included in the study. Patients with type 1 or secondary forms of diabetes mellitus, patients with severe hepatic or renal impairment, and patients with insulin and BMI 30 were excluded from the study. All the patients visiting diabetic center were reviewed on a daily basis and those who meet our study criteria were enrolled into the study, and informed consent was obtained from the subjects if he/she agrees 
to participate in the study. Demographics details, past medication history, and current treatment charts were recorded in data collection form. Baseline relevant investigations such as fasting blood sugar (FBS), PPBS (PPBS), and HbA1c were noted initially; patients were followed for next 3 months. FBS and Post Prandial Blood Sugar (PPBS) were reviewed in next three visits (each visit $30 \pm 5$ days), and HbA1c was rechecked only in the third visit to compare efficacy. Patients are also interviewed for any type of adverse reactions throughout the study. Based on medication received, patients were divided into three groups, Group 1 using biguanides (B)+sulfonylureas (SU), Group 2 on biguanides+thiazolidinediones (TZ), and Group 3 using biguanides+dipeptidyl peptidase inhibitors (DPI). Efficacy parameters (FBS, PLBS, and HbA1c) and Safety monitoring parameters are adverse drug reactions and body weight changes [at least 3\% of body weight change from baseline value] are compared in three visits. ANOVA was performed using SAS 9.1 to determine the level of significance in treatment groups before and after follow-up. The incidence rate was calculated to determine the burden of adverse drug reactions in treatment groups. Patient follow-up chart is given in Table 1.

\section{RESULTS}

During the study period, a total of 1530 patients were reviewed among them $180(11.76 \%)$ patients were excluded because type-1 diabetes, 224 (14.64\%) excluded because of sever comorbidities, and $730(64.88 \%)$ were excluded according to other exclusion criteria. Finally, 395 (25.81\%) were enrolled into the study. Among them, $273(69.11 \%)$ were male and $122(30.88 \%)$ were female.

The 395 Patients were distributed according to the oral hypoglycemic agents in prescription details are given in Table 2

About 42.02\% (166) patients were prescribed with the combination of biguanides + sulfonylurea followed by biguanides+TZ 34.93\% (138) and metformin+DPI were prescribed in $23.03 \%$ (91) patients.

FBS levels and PPBS levels were estimated in milligrams per deciliter (mg/dl) and calculated mean and standard deviation in all the three groups in each group and compare the FBS and PLBS using ANOVA test.

Evaluation and compare the FBS in the three groups and each visits FBS levels also given in detail in Table 3. In biguanides+DPI baseline, FBS level 177.2 \pm 33.23 (mg/dl), visit-1 FBS level $167.8 \pm 39.52$ (mg/dl),visit-2 FBS level 143.4 $\pm 35.05(\mathrm{mg} / \mathrm{dl})$, and visit-3 FBS level $128.1 \pm 25.43(\mathrm{mg} / \mathrm{dl})$ also statistically shown very

Table 1: Patients follow-up chart

\begin{tabular}{lllll}
\hline Monitoring Parameters & Visits & & & \\
\hline & Baseline & $\mathbf{1}$ & $\mathbf{2}$ & $\mathbf{3}$ \\
\hline FBS & $\sqrt{ }$ & $\sqrt{ }$ & $\sqrt{ }$ & $\sqrt{ }$ \\
PPBS & $\sqrt{ }$ & $\sqrt{ }$ & $\sqrt{ }$ & $\sqrt{ }$ \\
HbA1c & $\sqrt{ }$ & $\sqrt{ }$ & $\sqrt{ }$ & $\sqrt{ }$ \\
BMI & $\sqrt{ }$ & $\sqrt{ }$ & $\sqrt{ }$ & $\sqrt{ }$ \\
ADR identification & $\sqrt{ }$ & &
\end{tabular}

FBS: Fasting blood sugar, PPBS: Post Prandial Blood Sugar,

HbA1c: Glycosylated hemoglobin A1c, BMI: Body mass index,

ADRs: Adverse drug reactions

Table 2: Patients were distributed according to the oral hypoglycemic agents in prescription

\begin{tabular}{ll}
\hline Groups & $\begin{array}{l}\text { Number of } \\
\text { patients (\%) }\end{array}$ \\
\hline Biguanides+Sulfonylureas & $166(42.02)$ \\
Biguanides+Thiazolidinediones & $138(34.93)$ \\
Biguanides+Dipeptidyl Peptidase & $91(23.03)$ \\
Inhibitors & \\
\hline
\end{tabular}

highly significance from baseline to visit-3 ( $\mathrm{p}<0.0001)$, in biguanides + SU baseline FBS level 178.3 \pm 30.22 (mg/dl), visit-1 FBS level $164.4 \pm 39.92$ (mg/dl), visit-2 FBS level 152.2 $\pm 36.45(\mathrm{mg} / \mathrm{dl})$, and visit-3 FBS level $149.1 \pm 39.98$ (mg/dl) also statistically shown highly significance from baseline to visit- $3(\mathrm{p}<0.0019)$ and biguanides+TZ group baseline FBS level $176.4 \pm 29.56$ (mg/dl), visit-1 FBS level

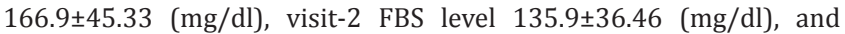
visit-3 FBS level $156.4 \pm 54.78$ (mg/dl) also statistically shown slightly significance from baseline to visit-3 $(\mathrm{p}<0.012)$. $\mathrm{p}$-value is found to be significant in all the three groups, but there is a greater significance in p-value of metformin+DPI $\left(<0.0001^{* * *}\right)$, followed by metformin+sulfonylurea $\left(0.0019^{* *}\right)$ and metformin+thiazolidinedione $\left(0.0127^{*}\right)$ are a little significant.

Evaluation and compare the postprandial blood sugar in the three groups and each visits postprandial blood sugar levels also given in detail in Table 4. In biguanides+DPI baseline PPBS level 266.2 \pm 53.12 (mg/dl), visit-1 PPBS level $232.4 \pm 66.48$ (mg/dl), visit-2 PPBS level 198.5 $\pm 64.01(\mathrm{mg} / \mathrm{dl})$, and visit-3 PPBS level $187.2 \pm 65.75(\mathrm{mg} / \mathrm{dl})$ also statistically shown highly significance from baseline to visit-3 ( $<<0.0062)$, in biguanides +SU baseline PPBS level 267.3 \pm 59.13 (mg/dl), visit-1 PPBS level $226.6 \pm 57.29$ (mg/dl), visit-2 PPBS level $215.6 \pm 64.28(\mathrm{mg} / \mathrm{dl})$, and visit-3 PPBS level $207.2 \pm 63.55(\mathrm{mg} / \mathrm{dl})$ also statistically shown slightly significance from baseline to visit-3 $(\mathrm{p}<0.026)$ and biguanides $+\mathrm{TZ}$ group baseline PPBS level 263.2 $\pm 47.11(\mathrm{mg} / \mathrm{dl})$, visit-1 PPBS level 230.3 \pm 73.05(mg/dl), visit-2 PPBS level 191.1 \pm 51.82 (mg/dl), and visit-3 PPBS level $206 \pm 63.28(\mathrm{mg} / \mathrm{dl})$ also statistically shown a little significance from baseline to visit-3 $(\mathrm{p}<0.022)$. $\mathrm{p}$-value is found to be significant in all the three groups, but there is a greater significance in p-value of metformin+DPI $\left(<0.0062^{* *}\right)$, followed by metformin+sulfonylurea $\left(0.026^{*}\right)$ and metformin+thiazolidinedione $\left(0.022^{*}\right)$.

Evaluation and compare the glycosylated hemoglobin (HbA1c) in the three groups detail given in Table 5 . In biguanides+DPI baseline HbA1c level $9.456 \pm 0.125(\%)$ and visit-3 HbA1c level 7.734 $\pm 0.865(\%)$ also statistically shown highly significance from baseline to visit- $3(\mathrm{p}<0.001)$, in biguanides +SU baseline HbA1c level 9.451 $\pm 0.152(\%)$ and visit-3 HbA1c level $8.936 \pm 1.536(\%)$ also statistically shown slightly significance from baseline to visit-3 $(\mathrm{p}<0.05)$, and biguanides $+\mathrm{TZ}$ group baseline HbA1c level 9.455 $\pm 0.187(\mathrm{mg} / \mathrm{dl})$ and visit-3 HbA1c level 8.914 $\pm 1.263(\%)$ also statistically shown a little significance from baseline to visit-3 $(\mathrm{p}<0.05)$. $\mathrm{p}$-value is found to be significant in all the three groups, but there is a greater significance in p-value of metformin+DPI $\left(<0.001^{* *}\right)$, followed by metformin+sulfonylurea $\left(0.05^{*}\right)$ and metformin+thiazolidinedione $\left(0.05^{*}\right)$ for safety purpose monitor the weight and adverse drug reaction and details were given in Tables 6 and 7.

In all the visits including baseline, patient weight was monitored and calculated BMI in $\mathrm{kg} / \mathrm{m}^{2}$. Details were given in Table 6. In biguanides+DPI baseline BMI $35.00 \pm 4.12(\mathrm{mg} / \mathrm{dl})$, visit-1 BMI $34.08 \pm 5.01(\mathrm{mg} / \mathrm{dl})$, visit-2 BMI $31.23 \pm 4.20(\mathrm{mg} / \mathrm{dl})$, and visit-3 BMI 30.76 $\pm 3.99(\mathrm{mg} / \mathrm{dl})$ also statistically shown highly significantly decreased from baseline to visit-3 ( $\mathrm{p}<0.0017)$, in biguanides +SU baseline BMI 31.45 \pm 1.32 (mg/dl), visit-1 BMI $31.62 \pm 1.85$ (mg/dl), visit-2 BMI 32.89 \pm 1.93 (mg/dl), and visit-3 BMI $32.01 \pm 2.00(\mathrm{mg} / \mathrm{dl})$ also statistically shown slightly significantly increased from baseline to visit- $3(\mathrm{p}<0.013)$ and biguanides+TZ group baseline BMI $32.12 \pm 2.11$ (mg/dl), visit-1 BMI 31.27 \pm 2.11 (mg/dl), visit-2 BMI $32.24 \pm 2.18$ (mg/dl), and visit-3 BMI $33.58 \pm 3.19$ (mg/dl) also statistically shown no significance from baseline to visit-3 $(\mathrm{p}<0.87)$ but value shown weight increased. P-value is found to be significant decrease biguanides+DPI $\left(p=0.0017^{* *}\right)$ and biguanides+SU group shown significantly increased the BMI ( $p, 0.0138^{* *}$ ) and $p$-value is found to be non-significant in biguanides+thiazolidinedione group (0.8741).

A total of 55 adverse drug reactions were identified in 55 (13.92) patients among 395 patients details were given in Table 7: In biguanides+SU group, $6.1 \%$ were experienced with hypoglycemia, it is only $1 \%$ in biguanides+DPI group and no hypoglycemia was identified into biguanides+TZ. Diarrhea 
Table 3: Comparison of FBS in three groups

\begin{tabular}{llllll}
\hline Group & Baseline FBS (mg/dl) & Visit 1 FBS (mg/dl) & Visit 2 FBS (mg/dl) & Visit 3 FBS (mg/dl) & p \\
\hline B+DPI & $177.2 \pm 33.23$ & $167.8 \pm 39.52$ & $143.4 \pm 35.05$ & $128.1 \pm 25.43$ \\
B+Su & $178.3 \pm 30.22$ & $164.4 \pm 39.92$ & $152.2 \pm 36.45$ & $149.1 \pm 39.98$ \\
B+Thi & $176.4 \pm 29.56$ & $166.9 \pm 45.33$ & $135.9 \pm 36.46$ & $0.0019 * *$ & $156.4 \pm 54.78$ \\
\hline
\end{tabular}

B+DPI=Biguanides+Dipeptidyl Peptidase Inhibitors, B+Su=Biguanides+Sulfonylureas, B+Thi=Biguanides+Thiazolidinediones. FBS: Fasting blood sugar

Table 4: Comparison of PPBS in three groups

\begin{tabular}{|c|c|c|c|c|c|}
\hline Group & Baseline PPBS (mg/dl) & Visit 1 PPBS (mg/dl) & Visit 2 PPBS (mg/dl) & Visit 3 PPBS (mg/dl) & $\mathbf{p}$ \\
\hline $\mathrm{B}+\mathrm{DPI}$ & $266.2 \pm 53.12$ & $232.4 \pm 66.48$ & $198.5 \pm 64.01$ & $187.2 \pm 65.75$ & $0.0062^{* *}$ \\
\hline $\mathrm{B}+\mathrm{Su}$ & $267.3 \pm 59.13$ & $226.6 \pm 57.29$ & $215.6 \pm 64.28$ & $207.2 \pm 63.55$ & $0.0263^{*}$ \\
\hline B + Thi & $263.2 \pm 47.11$ & $230.3 \pm 73.05$ & $191.1 \pm 51.82$ & $206 \pm 63.28$ & $0.0228^{*}$ \\
\hline
\end{tabular}

B+DPI = Biguanides+Dipeptidyl Peptidase Inhibitors, B+Su = Biguanides+Sulfonylureas, B+Thi = Biguanides+Thiazolidinediones. PPBS: Post prandial blood sugar

Table 5: Comparison of HbA1c in three groups

\begin{tabular}{lll}
\hline Group & Baseline HbA1c (\%) & Visit 3 HbA1c (\%) \\
\hline B+DPI & $9.456 \pm 0.125$ & p \\
B+Su & $9.451 \pm 0.152$ & $8.734 \pm 0.865$ \\
B+Thi & $9.455 \pm 0.187$ & $8.936 \pm 1.536$ \\
\hline B & $8.914 \pm 1.263$ & $<0.05^{*}$ \\
\hline
\end{tabular}

B+DPI=Biguanides+Dipeptidyl Peptidase Inhibitors, B+Su=Biguanides+Sulfonylureas, B+Thi=Biguanides+Thiazolidinediones. HbA1c: Glycosylated hemoglobin A1c

Table 6: Comparison of BMI in three groups

\begin{tabular}{|c|c|c|c|c|c|}
\hline Group & Baseline BMI $\left(\mathrm{kg} / \mathrm{m}^{2}\right)$ & Visit 1 BMI $\left(\mathrm{kg} / \mathrm{m}^{2}\right)$ & Visit 2 BMI $\left(\mathrm{kg} / \mathrm{m}^{2}\right)$ & Visit 3 BMI $\left(\mathrm{kg} / \mathrm{m}^{2}\right)$ & $\mathbf{p}$ \\
\hline $\mathrm{B}+\mathrm{DPI}$ & $35.00 \pm 4.12$ & $34.08 \pm 5.01$ & $31.23 \pm 4.20$ & $30.76 \pm 3.99$ & $0.0017^{* *}$ \\
\hline $\mathrm{B}+\mathrm{Su}$ & $31.45 \pm 1.32$ & $31.62 \pm 1.85$ & $32.89 \pm 1.93$ & $32.01 \pm 2.00$ & $0.0138^{* *}$ \\
\hline B+Thi & $32.12 \pm 2.11$ & $31.27 \pm 2.11$ & $32.24 \pm 2.18$ & $33.58 \pm 3.19$ & $0.8741(\mathrm{NS})$ \\
\hline
\end{tabular}

B+DPI=Biguanides+Dipeptidyl Peptidase Inhibitors, B+Su=Biguanides+Sulfonylureas, B+Thi=Biguanides+Thiazolidinediones. BMI: Body mass index

Table 7: Identification of ADR

\begin{tabular}{llll}
\hline ADR & B+Su n(\%) & B+THI n(\%) & B+DPI n(\%) \\
\hline Hypoglycemia & $8(6.1)$ & - & $1(1.09)$ \\
Diarrhea & $4(3.05)$ & - & $3(3.29)$ \\
Itching & - & - & $4(4.39)$ \\
Abdominal pain & $1(0.76)$ & $1(0.96)$ & $4(4.39)$ \\
Dizziness & $10(7.6)$ & $3(2.88)$ & $2(2.19)$ \\
Pedal edema & $1(0.76)$ & $4(3.84)$ & \\
Nausea & $2(1.52)$ & - & - \\
Vomiting & $2(1.52)$ & - & - \\
Indigestion & $2(1.52)$ & - & - \\
Cough & - & - & $1(1.09)$ \\
Total & $30(22.9)$ & $8(7.69)$ & $17(18.68)$ \\
\hline
\end{tabular}

ADRs: Adverse drug reactions

almost equally into biguanides+SU (3.05\%) and biguanides+DPI (3.29\%), which was not observed into biguanides+TZ. Itching was observed only into biguanides+DPI (4.39\%), abdominal pain also $4.39 \%$ were observed into biguanides+DPI, and $0.76 \%$ and $0.96 \%$ into biguanides+SU and biguanides+TZ, respectively. Dizziness was observed in all the three groups $7.6 \%, 2.88 \%$, and $2.19 \%$ in biguanides+SU group, biguanides+TZ, and biguanides+DPI, respectively. Pedal edema was reported $3.84 \%$ in biguanides+TZ and $<1 \%$ in biguanides+SU and vomiting, nausea, and indigestion were reported $1.52 \%$ in biguanides+SU.

\section{DISCUSSION}

Among 395 patients participated in the study, majority of them were male (69.11\%) followed by female (30.89\%), as known earlier, males are more likely to develop diabetes mellitus than women, which is similar to the study conducted by Gale and Gillespie [7]. This is because men are more susceptible than women to the consequences of laziness and obesity, possibly due to differences in insulin sensitivity and regional fat deposition.
This study demonstrates that there was a significant decline in FBS from baseline to end of the treatment of B+DPP group ( $p$ value $<0.001)$ when compared to $\mathrm{B}+\mathrm{SU}(\mathrm{p}=0.0019)$ and $\mathrm{B}+\mathrm{TZ}$ ( $\mathrm{p}$ value $0.0127)$ groups. Similarly, PLBS values showed significant decline in M+DPP group ( $p$ value 0.0062 ) than $M+S U$ ( $p$ value 0.0263 ) and $\mathrm{M}+\mathrm{TZ}(\mathrm{p}=0.0228)$ groups. HbA1c is also more significantly reduced in $\mathrm{M}+\mathrm{DPP}(<0.001)$ than in $\mathrm{M}+\mathrm{TZ}$ and $\mathrm{M}+\mathrm{SU}$ groups $(<0.05)$. This suggests that, DPI is superior to SU and thiazolidinedione's in glycemic control. According to a systematic review of 166 clinical studies, $\mathrm{M}+\mathrm{TZ}$ versus $\mathrm{M} /$ sitagliptin, M/SU versus M/DPP-4 inhibitor, and other combinations had similar efficacy at reducing HbA1c and blood glucose levels, which is slightly different from our study showing that $\mathrm{B}+\mathrm{DPP}$ is more effective than other combinations in reducing blood glucose levels.

Metformin appears to decrease the risk complications to overweight diabetic patients, and is associated with weight loss and fewer hypoglycemic attacks than are insulin and sulfonylureas; it is the first-line pharmacological therapy of choice of patients with diabetes mellitus. In this study, we assessed BMI changes into oral antidiabetic drugs when used in combination with metformin.

According to the study, there is a significant reduction of BMI after using $M+D P P(p=0.0017)$, which is similar to study conducted by Jennifer Green, which revealed that M+DPP used in type 2 diabetes, had potential weight loss effects as seen with metformin monotherapy [8]. A systematic review also showed that MET/DPP-4 inhibitors had a more favorable effect on weight, by reducing about $1.5-2.5 \mathrm{~kg}$. In our study, there is a significant increase in BMI, in patients using $\mathrm{M}+\mathrm{SU}$ $(p=0.138)$. According to the study conducted by Michael J. Fowler, patients using $\mathrm{M}+\mathrm{SU}$ experienced an increase $\geq 2 \mathrm{~kg}$ after initiation of these medications [9].

In patients using $\mathrm{B}+\mathrm{TZ}$, there is no significant change in $\mathrm{BMI}$ at the $3^{\text {rd }}$ visit. TZDs alone have potential to cause weight gain. With TZD 
treatment, there is a favorable shift in fat distribution of visceral to subcutaneous adipose depots that are associated with improvements in hepatic and peripheral tissue sensitivity to insulin [4].

However, according to systematic review, when $\mathrm{B}+\mathrm{TZ}$ combination is used, it reduces body weight by $0.9 \mathrm{kgs}$, which is slightly different from our study. Other adverse drug reactions reported in our study population are hypoglycemia, diarrhea, dizziness, nausea, vomiting, and indigestion, which are the highest in $\mathrm{M}+\mathrm{SU}$ group [10]. Pedal edema is the highest in $\mathrm{M}+\mathrm{TZ}$ group. Itching and abdominal pain are more in patients using M+DPP.

\section{CONCLUSION}

The study was conducted in 395 patients, of which 273 (69.11\%) were male and 122 (30.88\%) were female. A significant reduction in HBA1c \% was seen in all groups of patients. A significant reduction in BMI was seen in patients receiving DPI; while it was found to be increased form other group of patients. Adverse drug reactions observed were hypoglycemia, pedal edema, and itching attributed to metformin+sulfonylureas, metformin+pioglitazone, and metformin+DPI, respectively.

\section{REFERENCES}

1. Wild S, Roglic G, Green A, Sicree R, King H. Global prevalence of diabetes: Estimates for the year 2000 and projections for 2030. Diabetes
Care 2004;27:1047-53

2. Hollander P. Anti-diabetes and anti-obesity medications: Effects on weight in people with diabetes. Diabetes Spectrum 2007;20:159-65.

3. Golay A, Ybarra J. Link between obesity and Type 2 diabetes. Best Pract Res Clin Endocrinol Metab 2005;19:649-63.

4. Fonseca V. Effect of thiazolidinediones on body weight in patients with diabetes mellitus. Am J Med 2003;115 Suppl 8A:42S-8.

5. Pravinkumar VI, Talele GS. Comparative effects of metformin in combination with glimepiride and glibenclamide on lipid profile in Indian patients with Type 2 diabetes mellitus. Int J Pharm Pharm Sci 2011;3:472-4

6. Bonora E. Antidiabetic medications in overweight/obese patients with Type 2 diabetes: Drawbacks of current drugs and potential advantages of incretin-based treatment on body weight. Int J ClinPract Suppl 2007;154:19-28.

7. Gale EA, Gillespie KM. Diabetes and gender. Diabetologia 2001;44:3-15

8. Green J, Feinglos M. New combination treatments in the management of diabetes: Focus on sitagliptin-metformin. Vasc Health Risk Manag 2008;4:743-51.

9. Michael J, Fowler MD. Diabetes treatment, part 2: Oral agents for glycemic management. Clin Diabetes 2007;25:131-4.

10. Zaki NM, Maghrabi I. Trends in therapeutic interventions in patients with diabetes mellitus in Saudi Arabia. Int J Pharm Pharm Sci 2013;5:171-7. 